\title{
Simulation of lymph flow with consideration of natural gravity force influence
}

\author{
Anastasiia Mozokhina ${ }^{1, *}$ and Gennadii Lobov ${ }^{2, * *}$ \\ ${ }^{1}$ Lomonosov Moscow State University, 119991, Moscow, Russia \\ ${ }^{2}$ Pavlov Institute of Physiology, Russian Academy of Sciences, St. Petersburg 199034, Russia
}

\begin{abstract}
The model of lymph flow in the human lymphatic system in the quasi-one-dimensional approach is considered in the paper. Calculations with natural gravity force influence are presented. Calculations show that in some sets of parameters of lymphatic vessels the physiological flow can be reached in the model with zero global pressure gradient influence only by the "muscle" pump.
\end{abstract}

\section{Introduction}

The lymphatic system is a vessel system in the human body. It performs many functions, in particular, transport, drainage, immune, and depositing ones. In considering the transport function, LS consists of lymphatic vessels and lymph nodes. Lymphatic vessels have a specific structure, which provides the unidirectional flow of lymph: from the interstitial space to the upper vena cava. According to available scanty physiological observations, lymph flow in the LS is influenced by the pressure gradient, active and passive contractions of lymphatic vessels. Pressure in the interstitial space is close to zero, as well as pressure in the upper vena cava, so the global pressure gradient is small, can even be zero or negative. The pressure of lymph in the lymphatic vessels essentially is unknown. According to some sources, it can reach $20-40 \mathrm{~mm} \mathrm{Hg}$ in the thoracic duct [1]. Lymphatic vessels are divided by valves into segments which are called lymphangions. These lymphangions can produce active contractions. Active contractions with the prevention of backward flow of lymph by valves form a "muscle" pump, which is one more force propagating lymph in the LS. Active contractions have periodic - "phase" - part and tonic part, which is a reaction of the lymphatic vessel wall to the pressure difference in the lumen. Passive contractions are changes in the vessel lumen as a result of compression from surrounding tissues and the massage influence of big blood vessels. The degree of influence of each mechanism affecting lymph flow is not clear now, and we hope that current work can bring us closer to the understanding of mechanisms lying under lymph propagation in the human LS. Such understanding, in turn, can spread some light to the reasons for diseases related to the LS, for example, lymphedema.

\section{Model of lymph flow}

To get a model of lymph flow in the human lymphatic system in the quasi-one-dimensional approach, we created a graph of human LS. This graph is anatomically adequate, spatially

\footnotetext{
*e-mail: asm@cs.msu.ru

**e-mail: gilobov@yandex.ru
} 
oriented, consistent with a similar graph of the cardiovascular system [2], and is suitable for numerical calculations. The basis of the graph is regional groups of lymph nodes, which connected according to data from [3]. Arcs of the graph represent lymphatic vessels and lymph nodes, and vertexes of the graph represent points of vessel bifurcations, rare valves, and boundaries in the interstitial space and upper vena cava. All arcs of the graph are divided into four groups depending on mechanisms influencing lymph flow in the vessels of the group. These are big vessels with contractions, collectors with contractions and frequent valves, lymph nodes with contractions, and lymphatic capillaries, which are modeled by effective representations with no contractions and valves [4].

Lymph flow with no respect to contractions and frequent valves and respect to normal gravity force influence is modeled by a system of quasi-one-dimensional hemodynamic equations $[2,5]$ :

$$
\frac{\partial s}{\partial t}+\frac{\partial u s}{\partial x}=0, \frac{\partial u}{\partial t}+u \frac{\partial u}{\partial x}+\frac{1}{\rho} \frac{\partial p}{\partial x}=-8 \pi v \frac{u}{s}+g \cos \varphi, s=s(p)=s_{0}+\theta\left(p-p_{0}\right),
$$

where $x$ is a spatial coordinate, $t$ it time, unknown functions are velocity $u(x, t)$ and pressure $p(x, t), s(p)$ is a cross-sectional area which is a linear function of pressure $p, \rho$ is density, $v$ is viscosity, $g$ is a gravity factor, $\varphi$ is an angle between spatial axis of the vessel and gravity force vector. The third equation is so-called "state" equation, it contains all physiology. $s_{0}$ and $p_{0}$ are parameters of the vessel, $\theta$ describes elasticity of the vessel: the more $\theta$ is, the more elastic vessel is.

Flow in the vessel with frequent valves are modeled by changes in the right part of the second equation of (1):

$$
\frac{\partial u}{\partial t}+u \frac{\partial u}{\partial x}+\frac{1}{\rho} \frac{\partial p}{\partial x}=-8 \pi v(u) \frac{u}{s}+g \cos \varphi, v(u)=\frac{v_{\max }-v_{\min }}{\pi} \operatorname{arccot}(C u+D)+v_{\min } .
$$

Here $v$ is a function of velocity $u$ which gives normal value of viscosity $v_{\min }$ when lymph flows along with valves, and much more value $v_{\max }$ is the case of backward flow $[6,7]$.

Influence of contractions of the vessel are taken into account in the third equation of (1)

$$
s=s_{0}+\theta\left(p-p_{0}\right)+\theta A \sin \left(\frac{2 \pi}{\lambda}(x-a t)\right) .
$$

The third term is the periodic part of active contractions. This periodic part is modeled by a sine function, which is simple enough, still can give rich flow patterns, and so is widely used for such purposes [8-10]. Parameters are amplitude of the contraction $A$, wavelength $\lambda$, and velocity $a$. We also use frequency $\omega=2 \pi a / \lambda$ to describe contractions.

The graph is created and the following calculations are performed in the Cardio Vascular Simulation System (CVSS) [2]. To solve the nonlinear system (1) and its variations with equations (2) or (3) the finite difference schema with first-order time and second-order space approximation is used $[2,11-14]$.

\section{Calculations with natural gravity force influence}

\subsection{Test model}

The test model consists of one vessel placed in the gravity force. The vessel is vertically oriented in the way that gravity force influences in the direction against valves. We take the length of such vessel $200 \mathrm{~cm}$, so this is the simplest model of LS. Let us estimate pressure gradient needed to force lymph to flow against gravity force: $\mathrm{d} p=\rho g l=$ 
Table 1. Results of calculations of the test model

\begin{tabular}{lllllllll}
\hline No & description & 0 & 70 & 120 & 130 & 140 & 150 & 160 \\
\hline 1 & no valves & & & & -0.013 & & 0 & 0.006 \\
2 & $v_{\max }=4$ & & -0.0005 & 0 & 0 & & & \\
3 & valves type 1 & 0 & & & 0 & 0 & \\
\hline
\end{tabular}

$1 \mathrm{~g} / \mathrm{cm}^{3} \cdot 1000 \mathrm{~cm} / \mathrm{s}^{2} \cdot 200 \mathrm{~cm} \approx 150 \mathrm{~mm} \mathrm{Hg}$. So if we want lymph to flow against gravity force, the pressure gradient must be more then $150 \mathrm{~mm} \mathrm{Hg}$. Results of calculations (table 1, No 1) are consistent with this estimation, a positive value of flux is flow in the direction from interstitial space to upper vena cava against gravity force, a negative value is flow along with gravity force.

Other calculations in one vessel show that global $\mathrm{d} p$ can be reduced by valves. There are two types of valves in the model: valves in the big lymphatic vessels which lymphangions more than $1 \mathrm{~cm}$ (the first type, modeled by a condition of zero backward flux), and valves in vessels with lymphangions of 1-2 mm (the second type). With valves of the second type presented $\mathrm{d} p$ can be reduced to $120 \mathrm{~mm} \mathrm{Hg}$ (table 1, No 2), and it can be reduced to $0 \mathrm{~mm} \mathrm{Hg}$ in the case when valves of the first type are presented (table 1, No 3). Let us consider a model with the valve of the first type in the middle of the vessel. In this case, the pressure under the valve is $-74.2 \mathrm{~mm} \mathrm{Hg}$ and above the valve $p=76.2 \mathrm{~mm} \mathrm{Hg}$, so the pressure difference is $150.4 \mathrm{~mm} \mathrm{Hg}$ in the model, but now pressure in interstitial space and upper vena cava have physiological values $1 \mathrm{~mm} \mathrm{Hg}$.

As shown by calculations in the test model, valves can stop lymph from flowing down along with the gravity force, but cannot give a positive nonzero flux. Let us try to get a positive value of flux by adding contractions in the model.

Calculations are performed in one vessel with different length $l$. There are valves of type 2 with $v_{\max }=4 \mathrm{~cm}^{2} / \mathrm{s}$ and contractions with $A=0.1 \mathrm{~mm} \mathrm{Hg}, \lambda=1 \mathrm{~cm}$, and $a=1.5 \mathrm{~cm} / \mathrm{s}$ $(\omega=3 \pi)$, pressure is $1 \mathrm{~mm} \mathrm{Hg}$ in the boundaries. For $l=1 \mathrm{~cm}, l=10 \mathrm{~cm}$ and $l=100 \mathrm{~cm}$ time-average output flux is the same $y=-0.00035 \mathrm{ml} / \mathrm{s}$. These results show that length does not influence output flux, so further calculations are performed in the vessel of length $1 \mathrm{~cm}$.

The negative value of flux (time-average integral) shows, that lymph flows along with gravity force against valves. The positive value of flux can be got by alerting parameters of the vessel. Results of calculations with different parameters of the vessel and increasing efficiency of the "muscle" pump [15] are shown in table 2. By changing parameters of the vessel it is possible to get positive flux with $g=1000 \mathrm{~cm} / \mathrm{s}^{2}$ (table 2 , No 6-8, 11-16). The increase of $g$ leads to flux decrease. The increase of amplitude $A$ leads to the increase of output flux when parameters of the vessel stay the same (table 2, No 1, 6, 11-13, No 1416). Pressure gradient appearing in the vessels as a result of calculations less than $1 \mathrm{~mm} \mathrm{Hg}$. These calculations show that valves with contractions don't only stop lymph from flowing along with gravity force, but also give a nonzero output flux to the upper vena cava.

\subsection{Calculations on the graph}

Now we perform calculations on the LS graph (figure 1). Parameters of vessels are shown in table $3, p_{0}=0 \mathrm{~mm} \mathrm{Hg}$ for all arcs, $\theta$ from equation (3) has values $\theta_{1}$ from table 3 . Pressure in interstitial space is $3.4 \mathrm{~mm} \mathrm{Hg}$. Condition of free pressure is stated in the upper vena cava: $p^{\prime}=0$. There are valves of second type with $v_{\max }=4 \mathrm{~cm}^{2} / \mathrm{s}$, contractions with $A=0.1 \mathrm{~mm}$ $\mathrm{Hg}, \lambda=1 \mathrm{~cm}, a=1.5 \mathrm{~cm} / \mathrm{s}(\omega=3 \pi)$ and 25 valves of the first type in the model. Calculations in the horizontal position $(g=0)$ and vertical position $\left(g=1000 \mathrm{~cm} / \mathrm{s}^{2}\right)$ give us time-average integral fluxes $0.002 \mathrm{ml} / \mathrm{s}$ and $0.33 \mathrm{ml} / \mathrm{s}$ respectively. Flux in vertical case much more, then 
Table 2. Calculations of test model with different $\theta\left(\mathrm{cm}^{2} / \mathrm{mm} \mathrm{Hg}\right)$ and contraction parameters, 0 - 1000 are values of $g$ in $\mathrm{cm} / \mathrm{s}^{2}$. There are values of time-average integral flux in the cells under values of $\mathrm{g}$, $10^{-5} \mathrm{ml} / \mathrm{s}$. A (mm Hg) - amplitude of contractions, $\Delta s(\%)$ - difference in cross-section area, $d p$ $(\mathrm{mm} \mathrm{Hg})$ - pressure gradient, appearing in the system as a result of calculations

\begin{tabular}{lllllllllll}
\hline No & $\mathrm{A}$ & $\Delta s$ & $\theta$ & 0 & 400 & 500 & 600 & 700 & 1000 & $\mathrm{dp}$ \\
\hline 1 & 0.1 & 1.83 & 0.00165 & 7.8 & 1.1 & 0.3 & -0.4 & & -1.4 & 0.17 \\
2 & 0.1 & 1.81 & 0.00163 & 8 & & 0.2 & -0.5 & & -1.5 & 0.23 \\
3 & 0.1 & 0.34 & 0.00031 & 1 & & 0.1 & 0.02 & -0.005 & -0.08 & 0.17 \\
4 & 0.1 & 2.48 & 0.00223 & 13 & 0.05 & -1 & -3 & & -5 & 0.19 \\
5 & 0.1 & 2.65 & 0.00238 & 13 & 1 & -0.2 & & & -3 & 0.2 \\
6 & 0.2 & 3.67 & 0.00165 & 15 & & & & & 0.4 & 0.37 \\
7 & 0.2 & 3.63 & 0.00163 & 16 & & & & & 0.2 & 0.42 \\
8 & 0.2 & 0.68 & 0.00031 & 2 & & & & & 0.2 & 0.38 \\
9 & 0.2 & 5.3 & 0.00238 & 25 & & & & 3 & -0.6 & 0.26 \\
10 & 0.2 & 4.96 & 0.00223 & 26 & & & 3 & 2 & -3 & 0.36 \\
11 & 0.5 & 9.17 & 0.00165 & 32 & & & & & 14 & 0.56 \\
12 & 0.9 & 16.5 & 0.00165 & 55 & & & & & 31 & 0.63 \\
13 & 1 & 18.3 & 0.00165 & 59 & & & & & 35 & 0.73 \\
14 & 5 & 27.5 & 0.0005 & 150 & & & & & 120 & 0.7 \\
15 & 9 & 49.5 & 0.0005 & 250 & & & & & 230 & 0.86 \\
16 & 10 & 55 & 0.0005 & 280 & & & & & 250 & 0.81
\end{tabular}

flux in horizontal case, it happens because there is no control for total lymph volume in the model. Flux from head and neck grow with increase of $g$ and output flux grows as well.

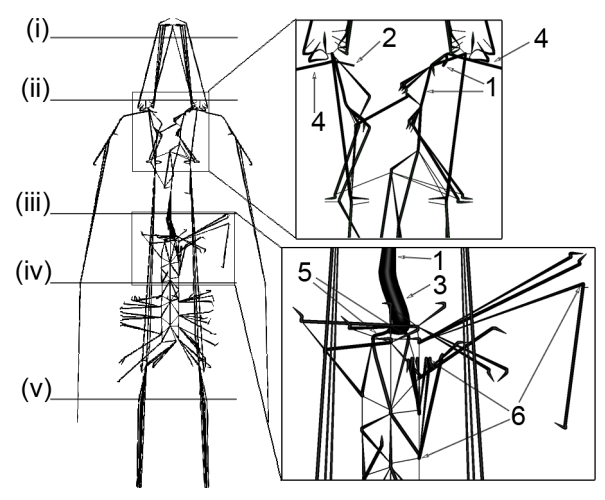

Figure 1. The graph of the lymphatic system: (i) — head, (ii) - neck, (iii) - diaphragm, (iv) elbows, (v) - groin; 1 - thoracic duct, 2 - right lymphatic duct, 3 - cisterna chyli, 4 - subclavian trunks, 5 - lumbar trunks, 6 - lymph nodes.

In the next set of calculations, we control input flux from the head and neck. Now the condition of constant input flux $q=1 \cdot 10^{-5} \mathrm{ml} / \mathrm{s}$ is stated in the inputs in the interstitial fluid of head and neck. There are $p=10 \mathrm{~mm} \mathrm{Hg}$ in other inputs, and $p=10 \mathrm{~mm} \mathrm{Hg}$ in upper vena cava. Valves of both types are included in the model, $v_{\max }=4$. Contractions have following parameters: $\lambda=1 \mathrm{~cm}, a=3 \mathrm{~cm} / \mathrm{s}(\omega=6 \pi)$. Parameters of the vessels are listed in table 3 , $\theta$ from equation (3) has values $\theta_{2}$ from table 3 . The results of the calculations are presented in figure 2 (circles). The increase in the amplitude of contractions $A$ leads to an increase in 
Table 3. Parameters of vessels of the graph

\begin{tabular}{llll}
\hline Vessel & $s_{0}, \mathrm{~cm}^{2}$ & $\theta_{1}, \mathrm{~cm}^{2} / \mathrm{mmHg}$ & $\theta_{2}, \mathrm{~cm}^{2} / \mathrm{mmHg}$ \\
\hline Cisterna chyli & 0.0503 & 0.02495 & 0.00468 \\
Thoracic duct & 0.0126 & 0.00623 & 0.00117 \\
Right lymphatic duct & 0.00316 & 0.00158 & 0.0003 \\
Lumbar trunks & 0.00708 & 0.00354 & 0.00066 \\
Other trunks & 0.00316 & 0.00158 & 0.0003 \\
Collectors & 0.0036 & 0.0015 & 0.00028 \\
Effective & 0.002 & 0.001 & 0.00019 \\
Lymph nodes & 0.0126 & 0.16457 & 0.03096 \\
\hline
\end{tabular}

output flux. The physiological value of flux $0.0352 \mathrm{ml} / \mathrm{s} \approx 3 \mathrm{l} /$ day is obtained with amplitude $A=3 \mathrm{~mm} \mathrm{Hg}$ which is $34 \%$ difference in diameter $(\Delta s=11.3 \%)$. The same quality results are obtained for the pressure $5 \mathrm{~mm} \mathrm{Hg}$ (figure 2, squares) and $3 \mathrm{~mm} \mathrm{Hg}$ (figure 2, triangles) in the interstitial space and upper vena cava. Decreasing of pressure in the interstitial space and upper vena cava (characteristic pressure in the calculation) leads to increasing of flux in the upper vena cava. The increase of pressure difference (by decreasing pressure in upper vena cava) also leads to an increase in output flux. Pressure gradient appearing as a result of calculations is shown in table 4 , it is $20-30 \mathrm{~mm} \mathrm{Hg}$ and maximum pressure reached in the calculations is about $11-25 \mathrm{~mm} \mathrm{Hg}$ (table 4 in parentheses).

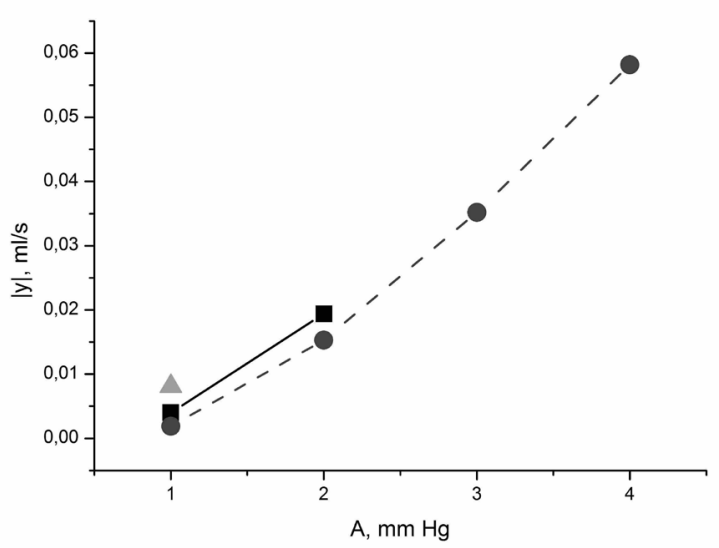

Figure 2. Results of calculations with natural gravity force influence with different characteristic pressures (in the interstitial space and upper vena cava, zero pressure gradient) and amplitudes of contractions. $A-$ amplitude of contractions, $|y|$ - average integral flux to the upper vena cava; squares characteristic pressure is $5 \mathrm{~mm} \mathrm{Hg}$, circles $-10 \mathrm{~mm} \mathrm{Hg}$, triangles $-3 \mathrm{~mm} \mathrm{Hg}$

\section{Conclusion}

Calculations on the graph show that the "muscle" pump (active contractions of the vessel with valves) can give a physiological value of output flux by itself in zero global pressure 
Table 4. Pressure appearing as a result of calculations in the LS graph with natural gravity force influence. $A(\mathrm{~mm} \mathrm{Hg})$ - amplitude of contractions, $p(\mathrm{~mm} \mathrm{Hg})$ - characteristic pressure in the calculation (initial pressure gradient is zero). There is resultant maximum pressure gradient $(\mathrm{mm} \mathrm{Hg}$ ) in the cells and values of maximum pressure $(\mathrm{mm} \mathrm{Hg})$ in the parentheses

\begin{tabular}{llll}
\hline$A$ & $p=10$ & $p=5$ & $p=3$ \\
\hline 1 & $25.7(17.19)$ & $20.27(11.19)$ & $25.5(14.7)$ \\
2 & $26.27(18.44)$ & $21.67(13.01)$ & \\
3 & $27.15(22.73)$ & & \\
4 & $27.88(25.26)$ & & \\
\hline
\end{tabular}

gradient. It means that active contractions can be the main force propagating lymph in the LS at least hydrodynamically.

\section{References}

[1] Petrenko V.M., Functional morphology of lymphatic vessels (DEAN, St. Petersburg, 2008) p. 400 (in Russian)

[2] Abakumov M.V., Gavrilyuk K.V., Esikova N.B., Lukshin A.V., Mukhin S.I., Sosnin N.V., Tishkin V.F., Favorskii A.P., Differential Equations 33, 895-901 (1997)

[3] Borzyak E.I., Bocharov V.Ya., Sapin M.R., Human anatomy (Medicina, Moscow, 1993) p. 560 (in Russian)

[4] Mozokhina A.S. and Mukhin S.I., Trends in Biomathematics: Modeling, Optimization and Computational Problems: Selected works from the BIOMAT Consortium Lectures (Springer, Cham, 2018) 325-334.

[5] Bunicheva A.Ya, Menyailova M.A., Mukhin S.I., Sosnin N.V., Favorskii A.P., Mathematical Models and Computer Simulations 5, 81-91 (2013)

[6] Simakov S., Gamilov T., Soe Y.N., Russian Journal of Numerical Analysis and Mathematical Modelling 28, 485-504 (2013)

[7] Mozokhina A.S., Mukhin S.I., Differential Equations 54, 938-944 (2018)

[8] Macdonald A.J., Arkill K.P., Tabor G.R., McHale N.G., Winlove C.P., Am. J. Physiol. Heart. Circ. Physiol. 295, 305-313 (2008)

[9] Bertram C.D., Macaskill C., Moore Jr.J.E., J. Biomech. Eng. 133, (2011)

[10] Rahbar E., Moore J.E.Jr., J. Biomech. 44, 1001-1007 (2011)

[11] Ashmetkov I.V., Khrulenko A.B., Mukhin S.I., Sosnin N.V., Favorskii A.P., Differential Equations 36, 1021-1026 (2000)

[12] Abakumov M.V., Esikova N.B., Mukhin S.I., Sosnin N.V., Tishkin V.F., Favorskii, A.P. Finite difference scheme for the solution of hemodynamic problems on the graph (DialogMSU, Moscow, 1998) p.16 (in Russian)

[13] Ashmetkov I.V., Mukhin S.I., Sosnin N.V., Favorskii A.P., Khrulenko A.B., Numerical investigation of properties of the finite difference scheme for hemodynamic equations (Dialog-MSU, Moscow, 1999) p.14 (in Russian)

[14] Mozokhina A.S., Mukhin S.I., Mathematical Models and Computer Simulations 11, 894-904 (2019)

[15] Mozokhina A.S., Mukhin S.I., Lobov G.I., Russ. J. Numer. Anal. Math. Modelling 34, 261-268 (2019) 